\title{
Erratum to: Comparison of Immigrant and Native-Born Population Adherence to Antipsychotic Treatment in a Spanish Health Region
}

\author{
Irene Forcada $\cdot$ Vanessa Pera $\cdot$ Inés Cruz $\cdot$ \\ Josep Pifarré $\cdot$ Catalina Serna $\cdot$ Montserrat Rué $\cdot$ \\ Leonardo Galván
}

Published online: 9 December 2012

(C) Springer Science+Business Media New York 2012

Erratum to: Community Ment Health J

DOI 10.1007/s10597-012-9551-9

The first and surnames of one of the authors were published erroneously. It should be Josep Pifarré instead of Pifarré Josep.

The online version of the original article can be found under doi:10.1007/s10597-012-9551-9.

I. Forcada · V. Pera

Psychiatry Unit, Hospital de Santa Maria, Avinguda Alcalde

Rovira Roure, 44, 25198 Lleida, Spain

I. Cruz $(\bowtie)$

Institut Català de la Salut, Unitat de Suport a la Recerca

Lleida-Pirineus, Institut Universitari d'Investigació

en Atenció Primària Jordi Gol, Barcelona, Spain

e-mail: icruzesteve@gmail.com

I. Cruz

Rambla de Ferran, 44, 38, 25007 Lleida, Spain

J. Pifarré

Hospital de Santa Maria, Institut de Recerca Biomèdica de

Lleida, Avinguda Alcalde Rovira Roure, 44, 25198 Lleida, Spain

C. Serna

Regional Primary Care Management Office, Institut Català

de la Salut, University of Lleida, Rambla de Ferran,

44, $3^{\circ}, 25007$ Lleida, Spain

M. Rué

University of Lleida, Montserrat Roig, 2, 25006 Lleida, Spain

L. Galván

Pharmaceutical Sciences, Pharmacy Unit of the Catalan Health

Department, Rovira Roure, 2, 25006 Lleida, Spain 